\title{
The Key Technology of Magnetic Bead Separation in the Flow Injection System
}

\author{
Wang Zhongsheng ${ }^{1}{ }^{*}$, Zhan Xin ${ }^{1}$, Wang Xuefeng ${ }^{2}$ \\ ${ }^{1}$ School of Computer Science and Engineering, \\ Xi' an Technological University, Xi' an 710021, P.R.C, \\ Email:wzhsh1681@163.com \\ ${ }^{2}$ Xi'an Yima Opto-Electronics Technology Co., Ltd.
}

\begin{abstract}
The magnetic bead separation system is a set of medical equipment which has a high degree of automation and separation. It has wide application prospect in our country, such as: Immunology, Cancer research, Transfer technique, Gene-technology, Histopathological, Clinical chemistry, Virology Microbiology and Medicine etc. This technique is mainly used for some kind of sample purification in some sectors( Hospital, Life science research center, Blood bank, drug test, Food inspection e.g. ), such as some kind of Bone marrow and Blood cells, Cancer cells, Nucleic acids, Bacteria and other Microbes, separation pool
\end{abstract}

\section{Keyword-magnetic bead, peristaltic pump driven}

\section{THE SELECTION OF MAGNETIC BEADS}

The large magnetic bead was selected, usually, when the abject have the characteristic of high concentration and low impurity. The diameters of large magnetic beads are usually from 1 to $10 \mathrm{um}$, and apt to precipitate. Large magnetic beads needs appropriate mixing to stay suspended in the sample. But when the object has low concentration, large magnetic beads are hardly to suspension and evenly distribution. At this time, we usually select the diameters of small magnetic beads between 50 to $200 \mathrm{~nm}$. Because of small magnetic beads can keep suspension and fully combine with object Rely on Brownian motion, so that we can to ensure the accuracy of trace analysis effectively. While, due to the weak magnetic of small magnetic beads, it is hard to recycle fully with simple device. And with the development of detection method, Medical testing on vitriol has higher and higher demand to the lower limit for the object. It also pushes the request more and higher to separate immune magnetic bend. But the current, our country is limited by technology. There is not any section to develop such high technique separation equipment, and then limit the development of medicine in vitriol immune detection technology, directly. We have large gap in the Chemiluminescence immunoassay technology and Electro encephalographic immunoassay technology, especially. According to this situation, we developed the flow injection magnetic bead separation system.

\section{THE TECHNOLOGY OF KEY SYSTEM}

\section{A. The peristaltic pump driven sampling system controlled by computer}

It can be make sure the number can be precise control, and insure quantitative analysis and detection for all kinds of simple. The precision of peristaltic pump is must be considered in the design. The important factor to Peristaltic pump sample is "the ripple effect". The process is as follows: fluid into the peristaltic pump's head and savings in the area which between the two sticks, so the formation fluid pillow. Followed by a short block and formation fluid pillow once again, because of behind a pump pipe was blocked again. Such cycles, then form a ripple rather than a smooth fluid pulsation. If this phenomenon can't be considered, it will have much impact on the accuracy of the sample. As shown in figure 1 .

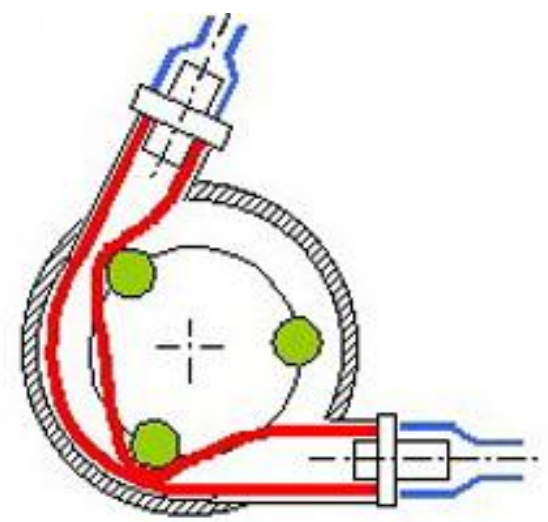

Figure 1. Peristaltic pump working principle diagram

Considering the effect, we should not only choose the pump which has low pulse but also to consider design a buffer device, when design a product. This kind of buffer device is belonging to the device that is reverse pump pipe extrusion. It can eliminate the pulse of the peristaltic pump with damping effect, through computer control the time and force of extrusion. In addition, the accuracy also relate to the precision of mechanical transmission system. Nonetheless, those errors have a track record of. We can correct it by software to make sure meet the requirements of precision.

The key technology project is involved as follows: 


\section{B. This magnetic bead separation pool which is a magnetic field lines flat and intensity of magnetic field can change}

In order to mark the different simple with diameter, we must using different intensity of magnetic field to separate these magnetic beads. For large magnetic beads, due to its quality magnetic is big. We can use smaller magnetic field intensity to separate it. Whole, small magnetic beads' quality magnetic is also small so that when we use the low intensity magnetic field they may be missed. Therefore it must be used which magnetic field intensity can be charge for these magnetic steel device. Besides, there is a magnetic field intensity extreme area, according to the ordinary magnetic lines of magnetic force distribution. In this area, the magnetic field intensity is the largest and easy to cause the magnetic beads piles, then making it difficult to dean. As a result, considering the structure of magnetic steel and the gap between magnetic steel and separation of the pool can effectively solve the problem when we design it. At the time of pilot study, we study this kind of be called "groove" magnetic. It is a kind of single magnetic field source is bonding on magnetic materials which have a u-shaped groove. In this kind of structure, magnetic material becomes an expansion through bond with the magnetic poles. As the pole "S" , extended around the pole "N", the magnetic field lines is dispersed and expanded to larger surface area which is form the magnetic field source. And due to one of the magnetic field source had been dispersing, the pole is extended. In this case, this is not applying to other magnetic field source, the direction of the magnetic field lines to be changed. This provides a flattened magnetic field. Under those decrease provides a larger area of an applied magnetic field. As shown in figure 2.

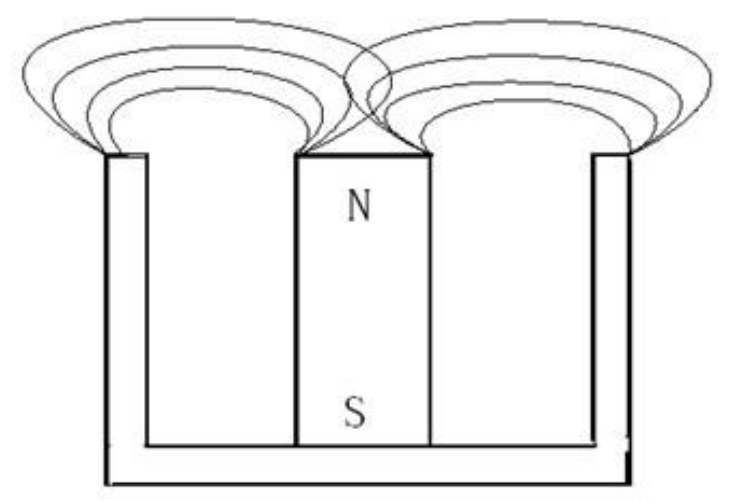

Figure 2. Composite magnetic steel structure diagram

\section{C. (3) The simple of automatic blending cultivation system}

In order to provide an appropriate environment and conditions to separate the test. We must to solve the problem what to deal with the simple automatically. For those reason, it should be through a progress which is marking the magnetic beads antibodies and combining with antigen, before we design a program to deal with the simple according automatically. How to write the system is also a key problem in this project. In a general way, using magnetic beads immune method to protein and gene, this process to be done automatically. Only in this way can ensure the accuracy after the separation.

In this project, we have designed a computer controlled processing units, the sample cup can rotate any direction and around the axis of rotation. And that sample processing units have vibration feature for the mixing of the sample. It equipped with a needle that can move along the designed trajectory. This needle is use for quantitative and configuration the sample liquid. As shown in figure 3.

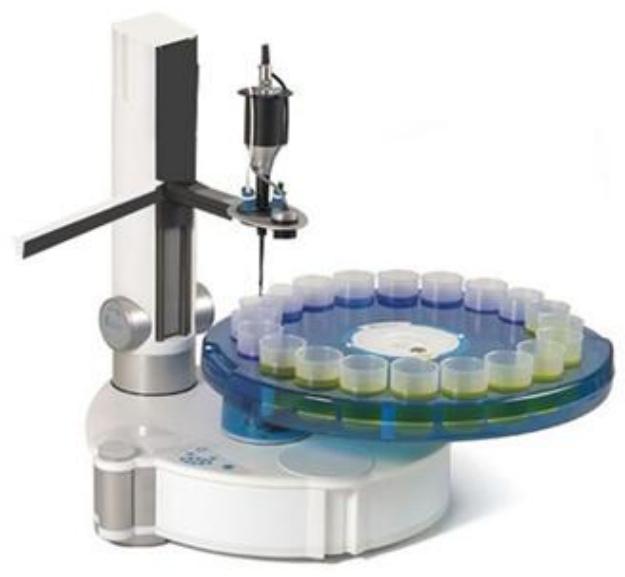

Figure 3. Sample processing units diagram

\section{The system of injection needle automatic flushing (Inside and outside flush)}

When we bulk separation of samples, often encounter the problem which is cross infection. Therefore, it is necessary to be considered that how to remove the flush on needle quickly and thoroughly. Firstly, we choose a special coating Teflon material which can solve the hang liquid problem. Secondly, how to design devices which can flush the sample needle automatically, it is the fourth key problem to solve in the process of project implementation.

\section{THE CHARACTERISTICS OF THE SYSTEM}

The flow injection immune magnetic bead separation system is based on analysis and research to the current international advanced equipment. A kind of method was summarized that is a new magnetic bead separation technology automatically. This method is according to the principle which magnetic particles and fluid separation to design a disk tubular magnetic beads flow separation pool. This kind of technology to improve the separation efficiency greatly, at the same time reduces the number of the separation of the sample greatly. This method is to separate the pool to provide a certain of speed fluid of separation, and it can increase 
the speed of the magnetic bead separation greatly, at the same time shorten the separation time. Under this premise which can insure the degree of separation and the stability, it would greatly reduce the cost of instrument manufacturing. In the development of the instrument, we apply a technique that is high precision flow injection automatically control. Makes the separation device to quantitative control in manage, sample injection and separation process. And laid a solid foundation for realize full automation.

\section{ACKNOWLEDGMENT}

This project is support by Xi'an Science and Technology Bureau.

Fond No.: CXY1351-7

\section{REFERENCES}

[1]Deshpande, Mangesh S, Kayatanavar, Devendra N, "FPGA implementation of AES encryption and decryption," International Conference on Control Automation. (2009)

[2]Yu-xin Wang, "Design and Implementation of USB Image Capture controller Based on FPGA," Xi'an University of Electronic Science and Technology. (2012)

[3]Fang Ren, Cheng-rui Yang, Lei-hua Chen, 3DES Implementation Based on FPGA, Journal of Xi' an Polytechnic University, vol. 25, 2011, pp. 555-559.

[4]Wei Yuan, The Design Principles of AES Algorithm and The Improvement of Its Key Expanding Algorithm, Jilin University. (2010)

[5]Kakarountas, Athanasios P, Goutis, Costas E, "A high-throughput area efficient FPGA implementation of AES-128 encryption," Workshop on Signal Processing Systems, IEEE. 2005, pp. 116-121.

[6]Jian-jun Guo, Chang-ming Liu, Yin Shi, Xia Lu, Jian Zhang, Hardware Implementation Analysis and Design of AES Algorithm. (2012)

[7] Huang Jian-lin, Ye Jian. Application of Fuzzy Self-tuning PID in the Temperature Control System for Tumor Hyperthermia[J].Research Work, 25 (2010) 41-43.

[8] Chen Ming-xin,Tang Ling-feng,Hu Jian.Research on Flow Control System of Pump Based on Fuzzy PID[J].Journal of Xinxiang University, 31 (2014) 36-40.

[9] Qi Lin.Research on Fuzzy and PID Dynamic Switching control Algorithm[D].Beijing Jiaotong University. (2008)

[10] Ying Wu, Hang Jiang, Min Zou.The Research on Fuzzy PID Control of the Permanent agnet Linear Synchronous Motor.Physics Procedia, 24 (2012) 1311-1318.

[11] Liu Xiao-feng,Liu Xiao-hui,Wang Long-shan,etc.Speed synchronization control of dual-motor in large crawler crane based on fuzzy PID control[J]. Journal of Jilin University(Engineering and Technology Edition), 41 (2011) 659-664.

[12] Onur Karasakal, Mujde Guzelkaya, Ibrahim Eksin, et,al.Online tuning of fuzzy PID controllers via rule weighing based on normalized acceleration[J]. Engineering Applications of Artificial Intelligence, 26 (2013) 184-197.

[13] Richa Sharma, K.P.S. Rana, Vineet Kumar.Performance analysis of fractional order fuzzy PID controllers applied to a robotic manipulator[J]. Expert Systems with Applications, 41 (2014) 4274-4289.

[14] Xie Guo-hua, Li Guo-dong,Liu Huan-jin, etc. MATLAB from Zero to Advanced[M]. Beijing University of Aeronautics and Astronautics. (2012) 